1 Edgarton AE. Herpes zoster ophthalmicus. Report of cases and review of literature. Arch Ophthalmol 1945;34:40-62.

2 Scheie GH, Alper MC. Treatment of herpes zoster ophthalmicus with cortisone or corticotropin. Arch Ophthalmol 1955;53:38-44.

${ }^{3}$ Marsh RJ. Herpes zoster keratitis. Trans Ophthalmol Soc UK 1973;93: 181-92.

4 Marsh RJ. Ophthalmic herpes zoster. Br f Hosp Med 1976;15:609-18.

(Accepted 5 August 1981)

Coventry and Warwickshire Hospital, Coventry CU1 4FH

ERNA E KRITZINGER, FRCS, MRCP, senior registrar in ophthalmology (present address: Birmingham and Midland Eye Hospital, Birmingham B3 2NS)

\section{Significance of early postoperative fever in children}

Fever is one of the earliest recorded signs of illness and has been a traditional observation in nursing care for centuries. A small rise in body temperature after operation is a well-recognised phenomenon, particularly in children, but its frequency and implication for care have never been assessed.

\section{Patients, methods, and results}

We studied 150 consecutive children (16 girls, 134 boys) undergoing operation for inguinal hernia. Three boys were also circumscised and two children underwent bilateral herniotomy. Age range was 4 months to 11 years (mean age 3.7 years). The procedure was planned on a day-case basis in all but 32 cases, and operations were performed by several different surgeons. All children were admitted on the morning of operation and recovered during the afternoon. During this period routine postoperative nursing observations were made, including at least two temperature record ings. The children were later seen by a doctor and allowed home at around $6 \mathrm{pm}$ if their condition was found to be satisfactory; if it was not they remained in hospital. Children were visited several times at home by the district nurse and reviewed in hospital at one month by the medical staff.

Body temperature above $37^{\circ} \mathrm{C}$ was often recorded, and the table shows a relation between temperature reached and age. The incidence of fever over $37.5 \mathrm{C}$ fell from $74 \%$ in those aged under 1 year to $28 \%$ in those over 4 years.

Proportion of children with fever after operation

\begin{tabular}{lccc}
\hline & \multicolumn{3}{c}{ Fever $\left({ }^{\circ} \mathrm{C}\right)$} \\
\cline { 2 - 4 } & $37-$ & $37 \cdot 5-$ & $\geqslant 38$ \\
\hline No (oo ${ }^{\circ}\left({ }^{\circ}\right)$ of children with fever within 24 hours & $83(55)$ & $71(47)$ & $33(22)$ \\
No $\left({ }^{\circ} 0\right.$ of children with fever at 6 pm & $66(44)$ & $56(37)$ & $32(21)$ \\
Average age (years) & 3.1 & $2 \cdot 9$ & 1.9 \\
\hline
\end{tabular}

Of 70 children with a temperature of over $37.5^{\circ} \mathrm{C}, 52$ were essentially well, six vomited transiently, and 12 developed complications possibly associated with fever (haematoma or infections). Two children without fever developed similar complications. All of the complications were apparent clinically, sometimes after discharge.

Of the 118 children for whom day-case treatment had been planned, 48 were detained overnight. In 28 cases this was solely because fever had been recorded; a similar number, however, were discharged despite fever. None of these was unwell. Nine of those detained stayed two or more days on account of unexplained fever. Pain, vomiting, or social reasons accounted for the remaining 20 who stayed overnight.

\section{Comment}

Temperatures over $37^{\circ} \mathrm{C}$ are conventionally considered to be abnormal. If only temperatures over $37.5^{\circ} \mathrm{C}$ are considered, however, $70(47 \%)$ of the 150 children studied were febrile within the first 24 hours. Interestingly, 12 of the 14 children who subsequently developed complications were from this febrile group. Complications were always apparent on clinical grounds, and decisions about management and discharge from hospital were never beneficially influenced by knowledge of the child's temperature. There therefore seems little useful predictive value in knowledge of fever in such cases.
The mechanism of the early postoperative rise in body temperature is not clear. A simple infective basis does not explain the relation above. There was no relation to postoperative chest infection. Excessive administration of atropine may result in fever, ${ }^{1}$ but there was no relation here to atropine dosage or other premedication. Hyperpyrexia occurring during anaesthesia is probably a completely different phenomenon, ${ }^{2}$ and no relation was noted here to the type of anaesthetic agent used. We suggest that the phenomenon may be a central response to the stress of surgery, entailing a transient adjustment of "set point" for temperature homoeostasis as occurs under other circumstances such as exercise. ${ }^{3}$ This would explain its lack of serious implication. Furthermore, the strong inverse relation to age suggests that it is a relatively primitive response. Whether the mechanism is reabsorption of blood (analogous to fracture fever) or other consequences of trauma inflicted remains speculative.

These observations have important implications for the management of operations done on a day-case basis. Even when only the temperature recorded at $6 \mathrm{pm}$ (the usual time of discharge) was considered, onethird of the patients were febrile $\left(>37.5^{\circ} \mathrm{C}\right)$. We suggest that temperature is of poor predictive value and may lead to unnecessary admission to hospital. It seems wiser in this type of operation to avoid measuring children's temperatures.

${ }^{1}$ Collins VJ. Principles of anaesthesia. Philadelphia: Lea and Febiger, 1976:1248.

${ }^{2}$ Atkinson RS, Rushman GB, Lee JA. A synopsis of anaesthesia. Bristol: Wright and Sons, 1977:653.

${ }^{3}$ Cooper KE. Regulation of body temperature $B r \mathcal{F}$ Hosp Med 1969;2: 1064-7.

(Accepted 28 August 1981)

Department of Surgery, Clinical Sciences Building, Royal Infirmary, Leicester LE2 7LX

IAN FRASER, FRCS, registrar

MICHAEL JOHNSTONE, FRCS, consultant

\section{Facial burns due to fan heater}

Facial heat injuries in toddlers are usually due to scalds. We describe a facial burn due to a fan heater, widely considered to be incapable of causing such injury.

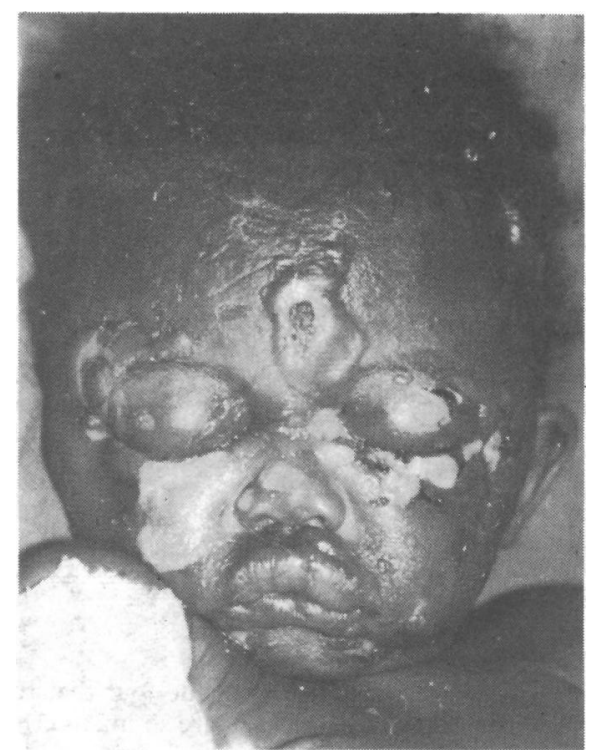

Facial appearance of child 12 hours after injury.

\section{Case report}

A 10-month-old West Indian girl who had been crawling for one month was left unattended on the floor with a 3-kW electric fan heater switched to maximum. She was discovered minutes later to have fallen over the front 